\title{
Myometrial Wound Healing Post-Cesarean Delivery in the MRL/MpJ Mouse Model of Uterine Scarring
}

\author{
Catalin S. Buhimschi, ${ }^{*}$ Guomao Zhao, ${ }^{*}$ \\ Nicoleta Sora, ${ }^{*}$ Joseph A. Madri, ${ }^{\dagger}$ \\ and Irina A. Buhimschi* \\ From the Departments of Obstetrics, Gynecology, and \\ Reproductive Sciences, ${ }^{*}$ and Pathology, ${ }^{\dagger}$ Yale University School of \\ Medicine, New Haven, Connecticut
}

There is little known about healing of the uterus after Cesarean delivery $(C D)$. Uterine wound repair was studied by using two strains of mice with different wound healing characteristics: $\mathrm{MRL} / \mathrm{MpJ}^{+/+}$(MRL: "high-healer" phenotype) and C57B1/6 ("low-healer" phenotype). First, we examined the morphology and histology of the uterine wall repair. We identified wound granulation tissue 3 days post-CD in both strains, albeit less in the MRL strain. Macroscopically, no scar could be identified either in MRL or C57B1/6 mice on day 60 post-CD. However, histologically, we found significant differences in wound integration, inflammation, and collagen birefringence between the two strains of mice. Using a histological index, we provided evidence for significant differences in mitotic activity in the initial phases of uterine healing among strains. Functional behavior of the uterine scar also was analyzed by using biomechanical parameters such as slope (measure of stiffness), yield point (measure of elasticity), and break point (measure of strength). There were significant differences in stiffness of the scarred myometrium between the two phenotypes. MRL mice displayed a significantly lower yield point compared with $\mathrm{C} 57 \mathrm{B1} / 6$. The break point was reached faster on days 15 and 60 in both C57B1/6 and MRL strains compared with day 3 postCD. Our findings indicate that differences in regenerative ability translate in histological, mitotic, and functional differences in biomechanical properties of the scarred myometrium after CD. (Am J Pathol 2010, 177:197-207; DOI: 10.2353/ajpath.2010.091209)

Cesarean delivery (CD) is the most frequently performed major surgical procedure in the industrialized world. By the latest national statistics it is reported that over $31 \%$ of women deliver by Cesarean section. ${ }^{1}$ In humans, difficulties in obtaining serial samples of the hysterotomy scar are a major barrier to our understanding of the events involved in the postpartum remodeling processes of the uterine wound post-CD. Thus, there is little known about healing of the uterine scar tissue after surgical injury. For example, whether the mammalian myometrium contain reserve cells that differentiate in myometrial myocytes to allow for scarless uterine regeneration remains largely unidentified. ${ }^{2,3}$

Wound healing of mammalian tissue is an essential process for maintaining organ integrity. The biological response to tissue injury in higher organisms falls into two main categories: repair and regeneration. ${ }^{4}$ In most mammals, the injured tissue is repaired by patching rather than restored to its original structure (ie, repair). Even when healing occurs with maximal efficacy, wound repair is dominated by a fibro-proliferative response and thus a fibrotic scar remains. ${ }^{5}$

Wound healing through regeneration is seen ancestrally in more primitive organisms (eg, amphibians). ${ }^{6}$ Interestingly, this type of wound healing has also been

Supported by National Institutes of Health grants RO3 HD 50249 (C.S.B.) and K12 HD 1027766 (Charles J. Lockwood, Yale Women's Reproductive Health Research Career Development grant)

C.S.B. and I.A.B. contributed equally, designed the study, performed the surgical procedures, collected the biological specimens, analyzed and interpreted the data, and drafted the article. N.S. participated in collecting and analyzing the biomechanical data. G.Z. performed the histological staining experiments. J.A.M. collaborated with C.S.B. and I.A.B. in designing the study and examining, quantifying, and interpreting the animal histological data. All the co-authors participated in formulating the hypothesis and had a significant contribution with aspects of study design, critical interpretation of the data, writing of the article, and approval of the final version.

Accepted for publication March 15, 2010.

Supplemental material for this article can be found on http://ajp. amjpathol.org.

Current address of N.S.: Division of Endocrinology, Department of Medicine, Medical University of South Carolina, Charleston, South Carolina

Address reprint requests to Catalin S. Buhimschi, M.D., Director Perinatal Research, Department of Obstetrics, Gynecology, and Reproductive Sciences, Yale University School of Medicine, 333 Cedar St, LLCl 804, New Haven, CT 06520. E-mail: catalin.buhimschi@yale.edu. 
reported in early gestation in mammalian fetuses and in adult rabbits. ${ }^{4,7}$ For instance, in humans, early in gestation, fetal wounds heal rapidly with minimal inflammation and complete regeneration of epithelial and mesenchymal tissues. The end result is a "scarless" healing with complete restoration of normal tissue architecture that is indistinguishable from normal tissue, whereas late in gestation fetal wound healing resembles that of adult mammals with fibrosis and scar formation. ${ }^{8}$ However, irrespective of the ultimate result, wound healing is a dynamic, interactive process involving mediators, blood cells, extracellular matrix, and parenchymal cells that follows three complex and overlapping phases: inflammation, tissue formation, and tissue remodeling. ${ }^{9}$

To provide a better understanding of the myometrial wound healing after CD, we turned our attention to an animal model of uterine wound scarring and repair. The $\mathrm{MRL} / \mathrm{MpJ}^{+/+}(\mathrm{MRL})$ mouse has proven to be an excellent model to study healing. ${ }^{10-13}$ Investigators have shown that punch holes made in the ears of MRL mice closed without scarring in 30 days ("high-healer" phenotype), whereas C57Bl/6 mice retained open ear holes for the rest of their lifetime ("low-healer" phenotype). ${ }^{11}$ A remarkable finding was that the ear-hole closure of the MRL not only displayed full healing but also showed recovery of normal tissue architecture, collagen orientation, normal angiogenesis, and appearance of hair follicles, sebaceous glands, and cartilage with lack of scar tissue. ${ }^{11}$ The unusual capacity of the MRL to heal was not a phenomenon unique to surgically induced skin wounds. In an animal model of cryogenically induced infarction of the heart, a key event was that the MRL myocardium healed with normal restoration of the ventricular wall structure and function. ${ }^{13}$ The biological response and functional recovery of the human myometrium after surgical injury are poorly understood. Yet, if the above observations extend to the uterus it is reasonable to consider that human uterine wound healing would be ideal if similar to the $\mathrm{MRL}$ rather than the $\mathrm{C} 56 \mathrm{BI} / 6$ healing phenotype.

The mechanisms responsible for the MRL's particular ability to heal continue to be subject of debate. ${ }^{14}$ The differential gene expression profile between MRL and C57BI/6 mice revealed that the fast wound ear repair of the MRL ("high healer") strain may be mediated by a metabolic shift toward a low inflammatory response. ${ }^{15,16}$ However, in a different study, Gourevitch et $\mathrm{al}^{12}$ showed that the regenerative response of the ear hole was characterized by a higher level of matrix metalloproteinase and a lower tissue inhibitor of metalloproteinase activity in the MRL compared with $\mathrm{C} 57 \mathrm{BI} / 6$ mouse. The enhanced matrix metalloproteinase healing response in the MRL was secondary to an increased neutrophil influx and thus inflammation at the site of injury. ${ }^{12}$ These findings are in contrast to the analysis of gene expression profile and suggest that further experimental research is necessary to understand the complexity of the regenerative process of the MRL mouse. ${ }^{16}$

In the present study we aimed to investigate for the first time the process of myometrial wound repair post-CD by using two strains of mice (MRL and C57BI/6) that differ significantly in their genetic background and underlying wound healing characteristics. We hypothesized that, compared with $\mathrm{C} 57 \mathrm{BI} / 6$, the higher regenerative capacity of the $M R L$ strain translates in enhanced myometrial wound healing, improved morphology, and functional behavior of the uterine scar.

\section{Materials and Methods}

\section{Mice}

$M R L$ and $\mathrm{C} 57 \mathrm{BI} / 6$ breeding pairs were purchased from Jackson Laboratory (Bar Harbor, ME) at 9 weeks of age. All animals were allowed free access to food and water and were exposed to standardized 12/12 hours daynight light cycles throughout the experiments. Our research and surgical protocols (number 2005-10966 and number A 01-02-02) were approved by the Institutional Animal Investigational Committee. Following establishment of our breeding colonies, we used time-pregnant nulliparous females (MRL $[n=18]$; C57BI/6 $[n=19]$ ).

\section{Surgical Procedures}

On day 17 of gestation (day 1 = sperm plug observed; day 19 = expected day of delivery) anesthesia was induced by using a combination of ketamine (60 to 80 $\mathrm{mg} / \mathrm{kg}$, Ketalar; Parke-Davis, Morris Plains, NJ) and xylazine (10 to 15 mg/kg, Gemini; Rugby Laboratory, Rockville Center, NY) administered intraperitoneal. ${ }^{17,18}$ Cesarean section was performed under sterile conditions. Once the abdomen was opened, a 3-cm longitudinal incision was made along the antimesometrial border in the mid-portion of each uterine horn. The fetuses and placenta were gently extruded through the hysterotomy. The uterine incision was closed by using one layer closure technique with continuous lock stitches of 5-0 polyglycolic acid suture (Supplemental Figure S1A, see http:// ajp.amjpathol.org). To mark the site of the hysterotomy, the edges of the uterine incision were marked with nonresorbable 4-0 prolene. In our laboratory the survival rate postsurgery reached $85 \%$. Animals were sacrificed at 3 days (C57BI/6: $n=4$; MRL: $n=5), 5$ days (C57BI/6: $n=5$; MRL: $n=5)$, 15 days (C57BI/6: $n=4$; MRL: $n=$ 4 ), and 60 days (C57BI/6: $n=5 ; \mathrm{MRL}: n=5)$ after surgery. Histological examinations were performed in all animals.

Ear punches (2-mm) were performed at the time of surgery in the center of the ear pinnae by using a mechanical caliper. ${ }^{10}$ A through-and-through wound was created. Diameters of the wound hole were measured post-CD and the ear-hole area (square millimeter) was calculated. Because we did not expect that either uterine tensile strength or size of the ear punches to change significantly from day 3 to day 5 post-CD, evaluation of these two parameters was performed only on days 3,15 , and 60 after surgery. 


\section{Bromodeoxyuridine Labeling of the Dividing Cells}

Bromodeoxyuridine (BrdUrd; 0.1\%; Sigma, St Louis, $\mathrm{MO}$ ), a marker for DNA synthesis and cell division was administered ad libidum in fresh drinking water starting with day 15 of pregnancy in all pregnant animals. ${ }^{13}$ The bottles containing BrdUrd water were protected from light and changed every 1 to 3 days. There were no ante- or postpartum animal side effects to BrdUrd administration.

\section{Histology}

We identified the site of the prior uterine injury (scar) between the nonresorbable 4-0 prolene markers and stain the site of uterine scar with the green tissue dye (Supplemental Figure S1B, see http://ajp.amjpathol.org) before tissue collection. The tissue scar was removed, fixed overnight in formalin (Fisher Chemical, Fairlawn, $\mathrm{NJ}$ ), and embedded in paraffin. Sections were cut to a thickness of $5 \mu \mathrm{m}$ and stained with H\&E. Tissue integration was defined as apposition of the surgical wound edges and was assessed as on a scale from 0 to 2 as absent (0), partial (1), or complete (2). Inflammation was characterized by the presence of polymorphonuclear leukocytes (PMNs), macrophages, and lymphocyte cells and graded on a scale from 0 (no reaction), 1 (low), 3 (moderate), or 4 (intense cellular infiltration). Grading of the tissue integration and inflammatory process was performed by two independent examiners (C.S.B. and I.A.B.).

Collagen birefringence, which represents an index of collagen fibers organization, was assessed in polarized light after staining with picosirius red. ${ }^{19}$ For each animal, digital images (original magnification, $\times 100$ ) were acquired by using an Olympus U-STP polarizing light microscope equipped with an Olympus OLY-200 digital camera (Olympus, Melville, NY).

\section{BrdUrd Detection and $\alpha$-Smooth Muscle Actin Double Staining}

Cellular proliferation was calculated by quantifying the rate of incorporation of the BrdUrd, a thymidine analog, into replicating DNA during the $S$ phase of the cell cycle. Uterine sections were cut to a thickness of $5 \mu \mathrm{m}$, mounted on coated glass slides, and processed as previously described. ${ }^{20}$ Sections of liver from each animal were used to confirm appropriate BrdUrd tissue levels. Briefly, after mounting, sections were permeabilized with $2.5 \%$ trypsin in phosphate buffer ( $\mathrm{pH} 7.4$ ) for 30 minutes. Sections were rinsed in PBS for five times and then incubated for 1 hour with $2 \%$ normal donkey serum to block nonspecific staining. DNA denaturation was achieved by immersion in $2 \mathrm{M} \mathrm{HCl}$ for 1 hour at $37^{\circ} \mathrm{C}$. Sections were incubated with primary monoclonal mouse anti-BrdUrd antibody (Zymed Laboratories, San Francisco, CA), diluted 1:1000 with PBS overnight at $4^{\circ} \mathrm{C}$. This was followed by wash in PBS and incubation for 1 hour with secondary biotinylated donkey anti-mouse immunoglobulin antibody
(Jackson ImmunoResearch, West Grove, PA), diluted 1:3000 with PBS. Immunohistochemical staining was performed with avidin-biotin staining (Elite ABC system, Vector Laboratories, Burlington, VT) with 3,3'-diamniobenzidine (Polysciences, Warrington, PA) as chromogen solution. Slides were counterstained with hematoxylin. Slides in which the first antibody was omitted served as negative control. The mitotic index was calculated as the percentage of BrdUrd positive nuclei from all nuclei counted in the field (original magnification, $\times 400$ ) in the area of the uterine scar site as identified by visible tissue dye. For each animal, the mitotic index values were averaged from five to six fields.

Double immunohistochemistry for BrdUrd and $\alpha$-smooth muscle actin ( $\alpha$-SMA) was performed sequentially by first detecting BrdUrD positive nuclei as described above except for using Nickel/3,3'-diamniobenzidine as substrate followed by additional blocking using UltraVision Block (Lab Vision Corporation, Fremont, CA). Incubation (1 hour at room temperature) with a mouse monoclonal primary anti- $\alpha$ SMA antibody (1:1000 dilution, Clone SM1, Thermo Scientific, Rockford, IL) was followed by development with biotinylated anti-mouse IgG (1:600, Jackson ImmunoResearch), horseradish peroxidase avidin-biotin complex (Elite ABC system, Vector Laboratories), and Vector NovaRED as a substrate. In initial experiments we optimized the concentration of each primary antibody. The specificity of immunostaining was validated with negative sections where the primary antibody has been preabsorbed with excess antigen in solution. Subsequently, negative control slides with omitted primary antibody were included with each run.

\section{Tensile Properties of the Uterine Scar}

We tested the tensile proprieties of the MRL and C57BI/6 scarred myometrium at 3,15 , and 60 days after CD. Rings of scarred myometrium were dissected, uniformly cut under microscope, and tested within 1 hour of collection. Given the significant involution of the uterus in postpartum, one ring per animal was tested. For consistency, the tested ring was from the middle of the left uterine horn in all animals. Tissues were anchored in a tissue bath containing $10 \mathrm{mmol} / \mathrm{L}$ HEPES/PBS solution $(\mathrm{pH}=7.4)$, by means of a silk thread passed through the lumen of each uterine ring. We used Shimadzu EZ-test instrumentation (Shimadzu North America, Columbia, $\mathrm{MD}$ ) to stretch the tissue by $0.42 \mathrm{~mm} /$ minute (sampling rate: $20 \mathrm{~Hz}$; duration of stretching: 0.7 seconds; duration or equilibration: 59.3 seconds). ${ }^{18,21}$ A representative stress-strain curve is shown in Supplemental Figure S2 (http://ajp.amjpathol.org). Parameters such as slope (measure of stiffness), yield point (moment when tissue changes its proprieties from elastic to plastic), and break point (BP; measure of tissue strength) were recorded and analyzed, as previously described. ${ }^{18,22}$ Once the intermittent stretching was initiated, it was continued past the yield point until the BP was reached. Slopes of the upper, lower, and mid-point regression lines were calculated to characterize the sample's resistance to stretch. A larger 


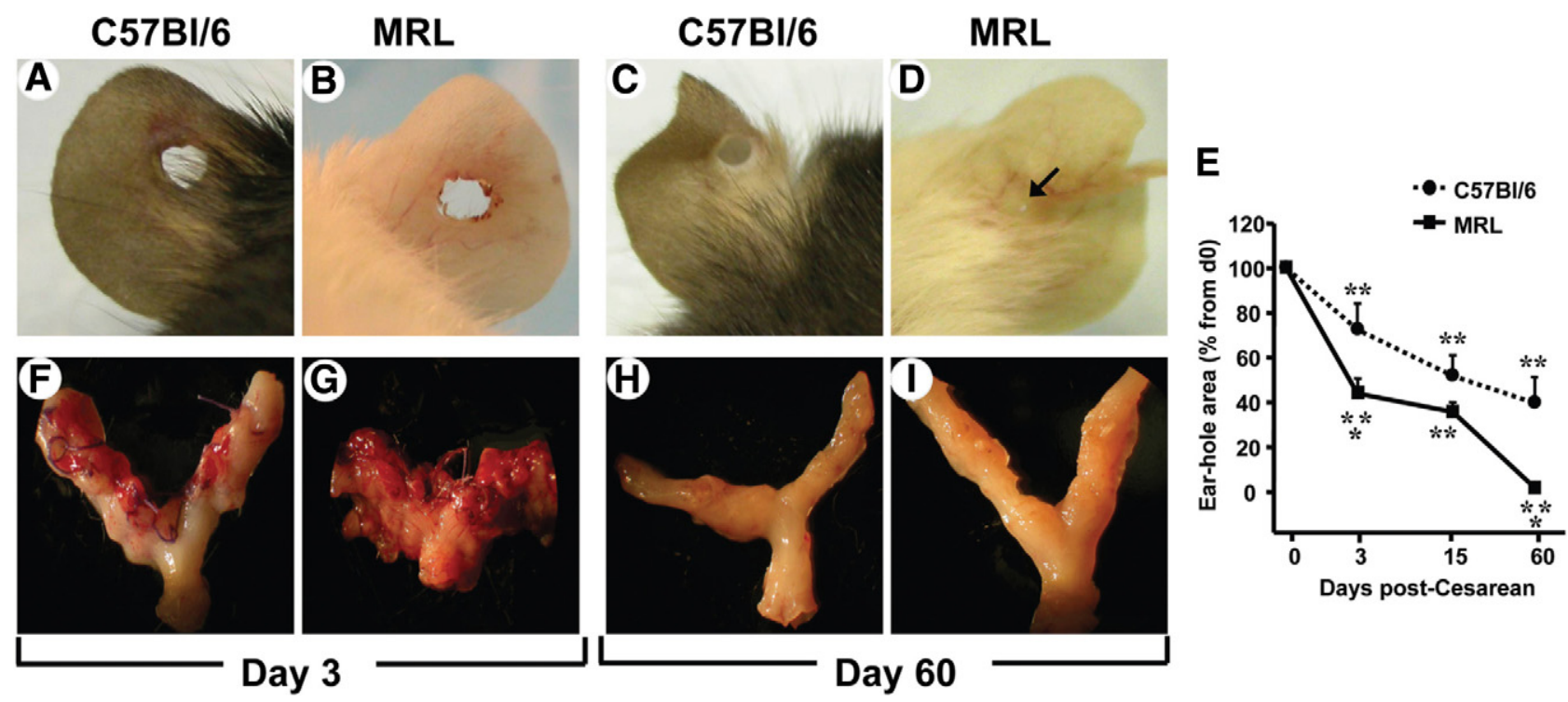

Figure 1. Concurrent macroscopic evaluation of the ear and uterine healing in the MRL/MpJ ${ }^{+/+}$(MRL) and $\mathrm{C} 57 \mathrm{Bl} / 6$ strains of mice. Representative pictures of the ears 3 days after surgery in the C57Bl/6 "low healer" (A) and MRL "high healer" (B) mouse are shown. Representative pictures of the ears 60 days after surgery in the $\mathrm{C} 57 \mathrm{Bl} / 6$ (C) and MRL (D) mouse are shown. The arrow in $\mathbf{D}$ points to the remaining lumen of the ear-hole in an MRL mouse at 60 days after puncture $\mathbf{E}$ displays the percent change in ear-hole area postinjury. Statistical analysis: two-way analysis of variance followed by posthoc Student-Newman-Keuls tests; ${ }^{*} P<$ 0.01 versus initial ear punch area (day 0$) ;{ }^{*} P<0.01$ versus $\mathrm{C} 57 \mathrm{Bl} / 6$ phenotype. Representative pictures of the uterus three days post cesarean in $\mathrm{C} 57 \mathrm{Bl} / 6(\mathbf{F})$ and MRL (G) mouse are shown. Representative pictures of the uterus 60 days postcesarean in the C57Bl/6 (H) and MRL (I) mouse are shown.

slope indicates a higher resistance to stretch, and therefore increased stiffness. ${ }^{22}$ Data were normalized to the dry weight of the myometrial tissue. We analyzed the location of uterine rupture and noted whether disruption of the tissue occurred at the scar's site or not.

\section{Statistical Analysis}

The normality of the data distribution was tested by using the Kolmogorov-Smirnov test. Analysis was performed with the aid of SigmaSTAT 2.03 statistical software (Jandel Corporation, San Rafael, CA). The data are presented as their mean and SEM. Comparisons between groups included one-way or two-way analysis of variance, as appropriate. Although the arithmetic means are reported in the text, statistical testing by using two-way analysis of variance was performed following logarithmic transformation of the data. Differences in proportions were examined with Fisher's exact test. Four to five animals per group confers sufficient power to identify 50\% differences in slope between the MRL and C57BI/6 strains (power $=0.8 ; \alpha=0.05$ ) in two-way analysis of variance. A $P<0.05$ was considered to indicate statistical significance.

\section{Results}

\section{Ear Healing}

To confirm that the MRL and C57BI/6 mice have different healing phenotypes as earlier reported for animals outside pregnancy, ${ }^{10}$ we performed ear punches at the time of CD. We confirmed that postpartum the ear of the white coat $M R L$ mice healed more rapidly than of the black coat
C57BI/6 controls (Figure 1). Compared with C57BI/6 (Figure $1 \mathrm{~A})$, granulation tissue was present 3 days postear punch in the MRL strain (Figure 1B). Sixty days postsurgery, the ears of the C57BI/6 maintained an open earhole (Figure 1C), whereas the ears of MRL mice most frequently healed completely and without scarring (ie, by regeneration; Figure 1D). In Figure $1 \mathrm{E}$ we display the change in ear-hole area of C57BI/6 and MRL mice after surgery. Significant differences between the high and the low healer strains of mice were registered at 3 (two-way analysis of variance, $P=0.006)$ and 60 days $(P<0.001)$ postsurgery.

\section{Macroscopic Aspect of Uterine Healing}

Lax adhesions were observed at 3 and 5 days post-CD in $50 \%$ of $\mathrm{C} 57 \mathrm{BI} / 6$ and all MRL mice. At 15 days, we observed tight adhesions of the mesenterium to the uterine surface in all animals in both strains. Interestingly, at 60 days these adhesions became lax again in the MRL animals and in the C57BI/6 strain disappeared entirely. In Figure 1, F-I, we show the external aspect of the uterus in the MRL and C57BI/6 strain of mice on day 3 and day 60 postsurgery. Although more in the MRL strain, abundant granulation tissue was identified at the surface of the uterine scar 3 days post-CD in both strains of mice (C57BI/6: Figure 1F; MRL: Figure 1G), and the surgical stitches appeared loose. On day 5, granulation tissue buds were still present in $\mathrm{C} 57 \mathrm{BI} / 6$ but not in the MRL mice. By day 15, the granulation tissue disappeared although the site of the uterine scar could be identified macroscopically in both strains. By contrast, at day 60 postsurgery no scar could be easily identified in either the $\mathrm{C57BI} / 6$ (Figure 1H) or MRL (Figure 1I) mice. Preser- 
Bar $\quad$ C57BI/6
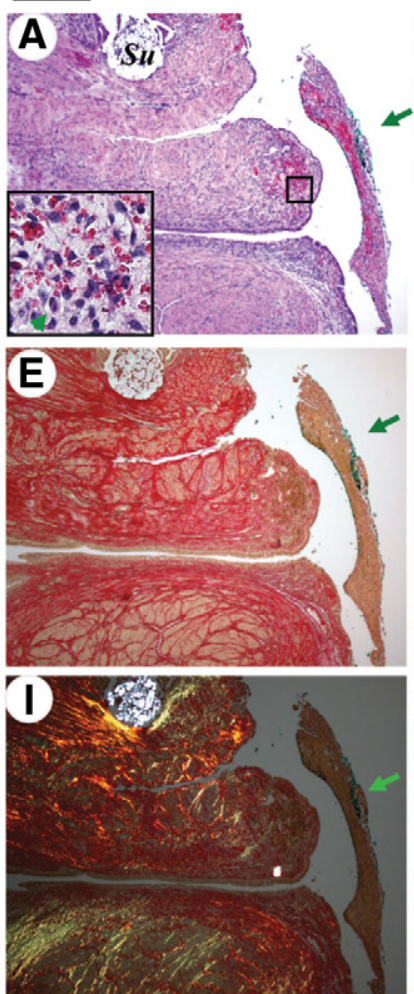

Day 3
MRL
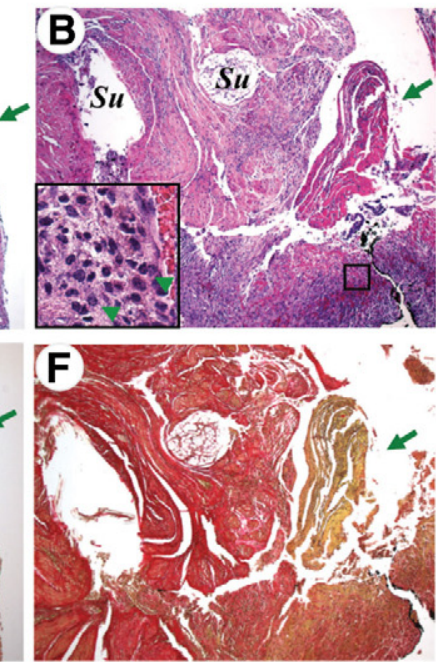

(J)
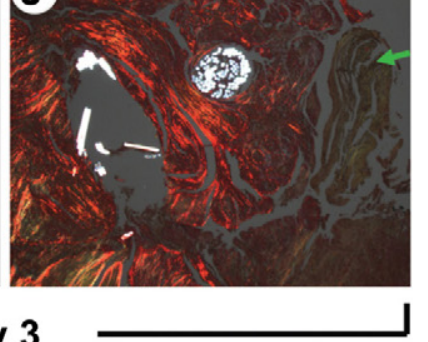

C57BI/6
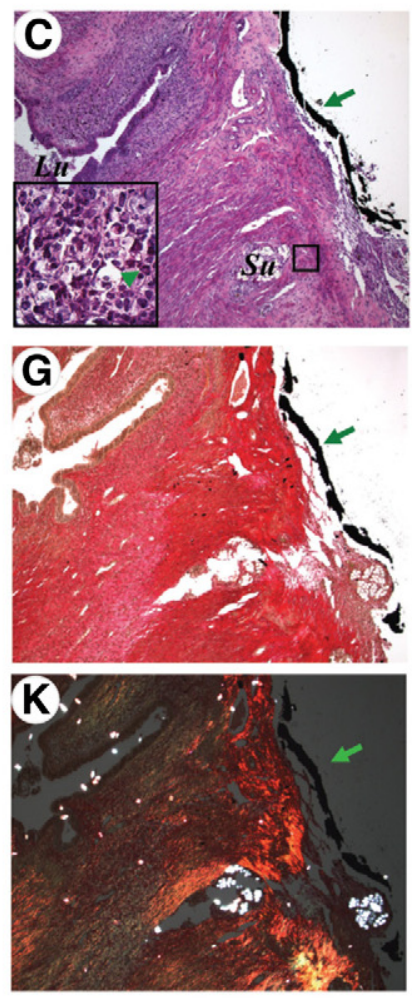

Day 5
MRL
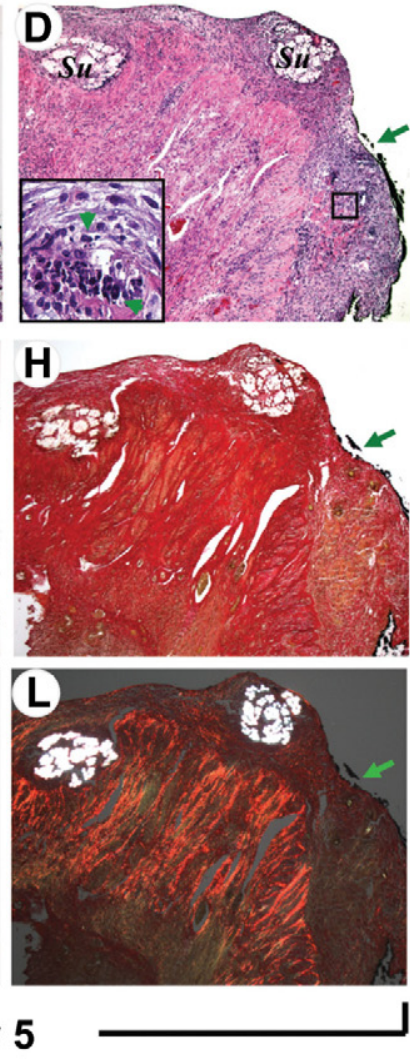

Figure 2. Representative histological sections of the uterine scar site on days three and five post-Cesarean delivery in $\mathrm{C} 57 \mathrm{Bl} / 6$ and $\mathrm{MRL} / \mathrm{MpJ}{ }^{+/+}$(MRL) mice are shown. Adjacent sections were stained with either H\&E [C57Bl/6: day three (A) and day five (C) versus MRL: day three (B) and day five (D)] and Sirius red visualized in either white light $[\mathrm{C} 57 \mathrm{Bl} / 6$ : day three $(\mathbf{E})$ and day five $(\mathbf{G})$ versus MRL: day three (F) and day five (H)] or polarized light $[\mathrm{C} 57 \mathrm{Bl} / 6$ : day three (I) and day five5 (K) versus MRL: day three ( $\mathbf{J}$ ) and day five $(\mathbf{L})$ ]. The green arrowheads in the insets mark PMNs. The green arrows outside the insets indicate the site of the uterine scar as marked with tissue dye (green or black). The round intensely birefringent multilocular structures are the Vicryl multifilament surgical sutures (Su; marked only on the top panels). Lu marks the uterine lumen. Original magnification, $\times 100$ (Scale bar $=200 \mu \mathrm{m})$. The small squares on the H\&E panels mark the area shown magnified (Scale bar $=40 \mu \mathrm{m}$ ) in the inset of the corresponding panel.

vation of a patent uterine lumen was confirmed in both strains at 60 days postsurgery.

\section{Histological Examination of Uterine Healing}

We identified the site of prior uterine surgical injury in both strains of mice at the site marked by the tissue dye. In Figure 2 we show representative histological images of the uterine surgical site at days 3 and 5 post-CD. Illustrative histological images of the uterine wall 15 and 60 days post-CD are shown in Figure 3.

\section{Wound Integration}

On day 3 postsurgery, wound integration was absent in all C57BI/6 mice (four of four; Figure 2A), whereas two of five $M R L$ mice had partial apposition of wound edges (Figure 2B). By day 5, four of five C57BI/6 mice had partial integration (Figure $2 \mathrm{C}$ ). On day 5, all MRL mice (five of five) had partial integration, whereas 2 of 5 animals had already complete surgical wound integration (Figure 2D). On days 15 (Figure 3, A and B) and 60 (Figure 3, C and D), integration appeared complete in both strains. Analysis of the semiquantitative scoring system demonstrated significant differences in the healing process, which was impacted both by the days post-CD (two-way analysis of variance, $P<0.001$ ) and genetic strain $(P=0.013)$. We observed an accelerated integration in the MRL compared with $\mathrm{C} 57 \mathrm{BI} / 6$ mice $(P=0.048$ for interaction between days and strain). See also Supplemental Figure S3A available at http://ajp.amjpathol.org.

\section{Wound Inflammation}

H\&E staining demonstrated presence of an inflammatory infiltrate involved in the reparative myometrial processes in both $\mathrm{C} 57 \mathrm{BI} / 6$ and MRL mice. Judging from the number of cells per field, 3 days postsurgery the most prominent initial inflammatory response was noticed in the MRL strain at the site of uterine wound as seen in Figure $2 \mathrm{~B}$ versus Figure 2A. In the MRL animals, in the early inflammatory phase (day 3), buds of granulation tissue were observed also within the uterine lumen (not shown). This was not present in C57BI/6 animals, suggesting that the early inflammatory process may be more prominent in $M R L$ mice. The semiquantitative analysis of the inflammatory infiltrates demonstrated the most conspicuous dif- 

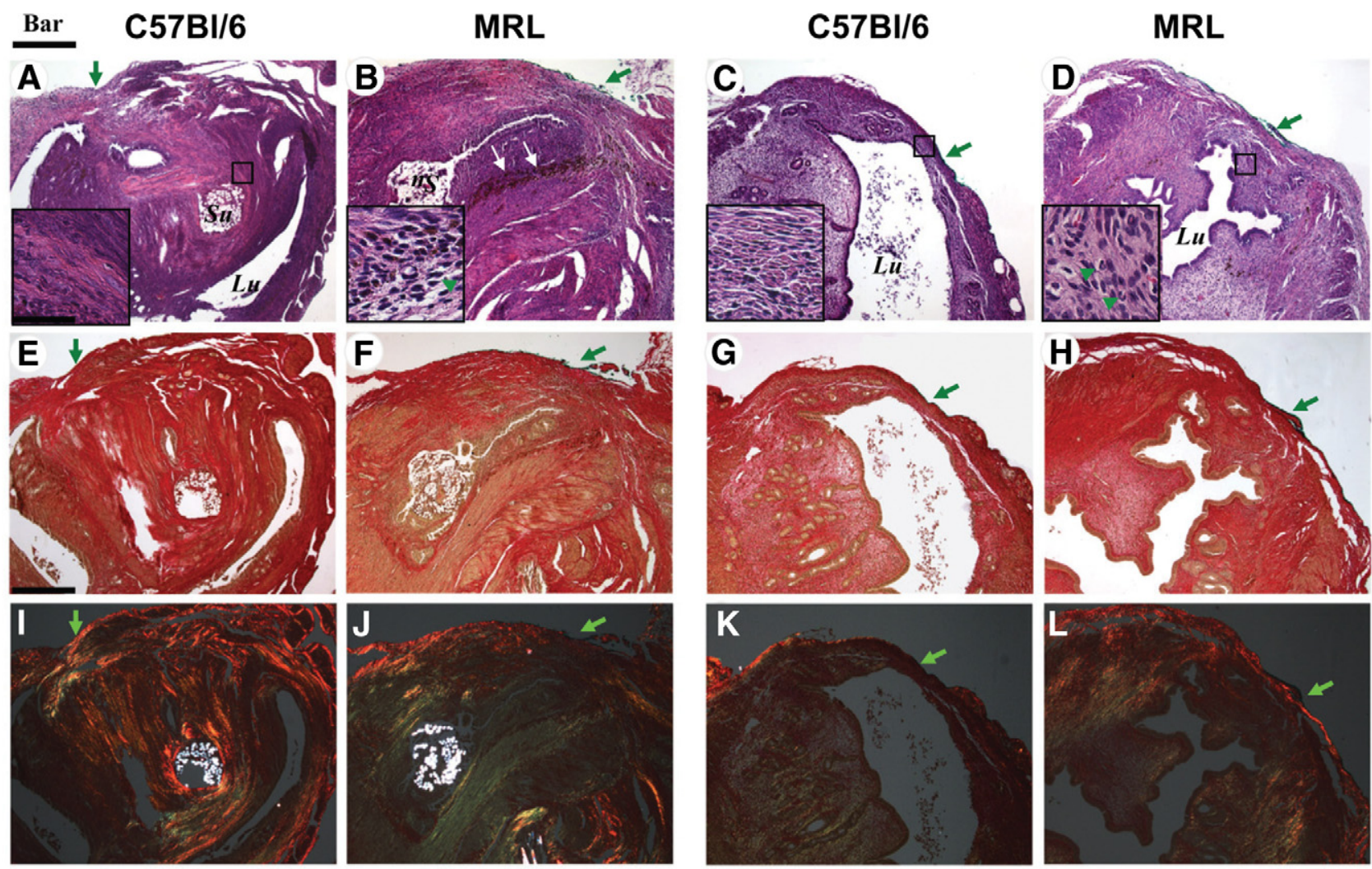

Day 15

Day 60

Figure 3. Representative histological sections of the uterine scar site on days 15 and 60 post-Cesarean delivery in $\mathrm{C} 57 \mathrm{Bl} / 6$ and $\mathrm{MRL} / \mathrm{MpJ}{ }^{+/+}$(MRL) mice are shown. Adjacent sections were stained with either H\&E [C57Bl/6: day $15(\mathbf{A})$ and day $60(\mathbf{C})$ versus MRL: day $15(\mathbf{B})$ and day 60 (D)] and Sirius red and visualized in either white light [C57Bl/6: day $15(\mathbf{E})$ and day $60(\mathbf{G})$ versus MRL: day $15(\mathbf{F})$ and day $60(\mathbf{H})]$ or polarized light [C57Bl/6: day $15(\mathbf{I})$ and day $60(\mathbf{K})$ versus MRL: day $15(\mathbf{J})$ and day $60(\mathbf{L})$ ]. The green arrowheads in the insets mark PMNs. The green arrows outside the insets indicate the site of the uterine scar as marked with tissue dye (green or black). The white arrows in $\mathbf{B}$ point to hemosiderin deposits in the area of integration. The round intensely birefringent multilocular structures are the Vicryl multifilament surgical sutures (Su; marked only on the top panels). Lu marks the uterine lumen. Original magnification, $\times 100($ Scale bar $=200 \mu \mathrm{m})$. The small squares on the H\&E panels mark the area shown magnified $($ Scale bar $=40 \mu \mathrm{m})$ in the inset of the corresponding panel.

ference between $\mathrm{MRL}$ and C57BI/6 mice on day 5 postsurgery (two-way analysis of variance, $P=0.001$; see also Supplemental Figure S3B available at $h$ ttp:// ajp.amjpathol.org). The infiltrates were more abundant in the subserosal area and around sutures. In both strains the mixed inflammatory infiltrate consisted of PMNs identified by the multilobulated aspect of the nucleus (marked with green arrowheads in high magnification inserts), plasma cells, and activated monocytes. By day 15, in C57BI/6 mice the predominant cell population in the scar area were activated fibroblasts judged by their vesicular nucleus (Figure 3A, insert). Instead, the inflammatory process persisted in MRL mice (Figure 3B). On day 60 we could not identify the inflammatory infiltrates any longer in C57BI6 mice (Figure 3C), whereas in the MRL, scattered PMNs could still be observed (Figure 3D, insert, green arrowheads).

\section{Collagen Birefringence at the Site of Uterine Scar}

Analysis of collagen deposition (days 3 and 5) by picrosirius red in white light (Figure 2, E-H) and polarized light microscopy (Figure 2, I-L) showed that in both strains of mice the site of the myometrial wound was not individualized as a distinct area of collagen deposition before day 15 postsurgery. At this stage (day 15: Figure 3, I-L), polarized microscopy showed that birefringent collagen was present at the site of the previous hysterotomy in both strains. The site of the myometrial wound was covered by fibrinous exudate, which was better organized compared with day 3 post-CD for both strains of mice. Overall, beginning with day 15, the tissue architecture appeared better organized in the MRL mice (Figure 3J) compared with $\mathrm{C} 57 \mathrm{BI} / 6$ strain (Figure $3 \mathrm{I}$ ). In the MRL mouse picrosirius red staining could not identify any areas of mature collagen condensation (Figure 3, F and J), whereas in the $\mathrm{C} 57 \mathrm{BI} / 6$, orange-red birefringent collagen was more prominent especially in the areas surrounding the surgical stitches (Figure 3, E and I). Brownish pigments like lipofuscin and hemosiderin were visible at the area of integration in both $\mathrm{MRL}$ and $\mathrm{C} 57 \mathrm{BI} / 6$. A representative image is shown for the $M R L$ in Figure $3 B$ (white arrows).

On day 60 , the $M R L$ wound integration was complete and the uterine architecture was restored to normal (Fig- 

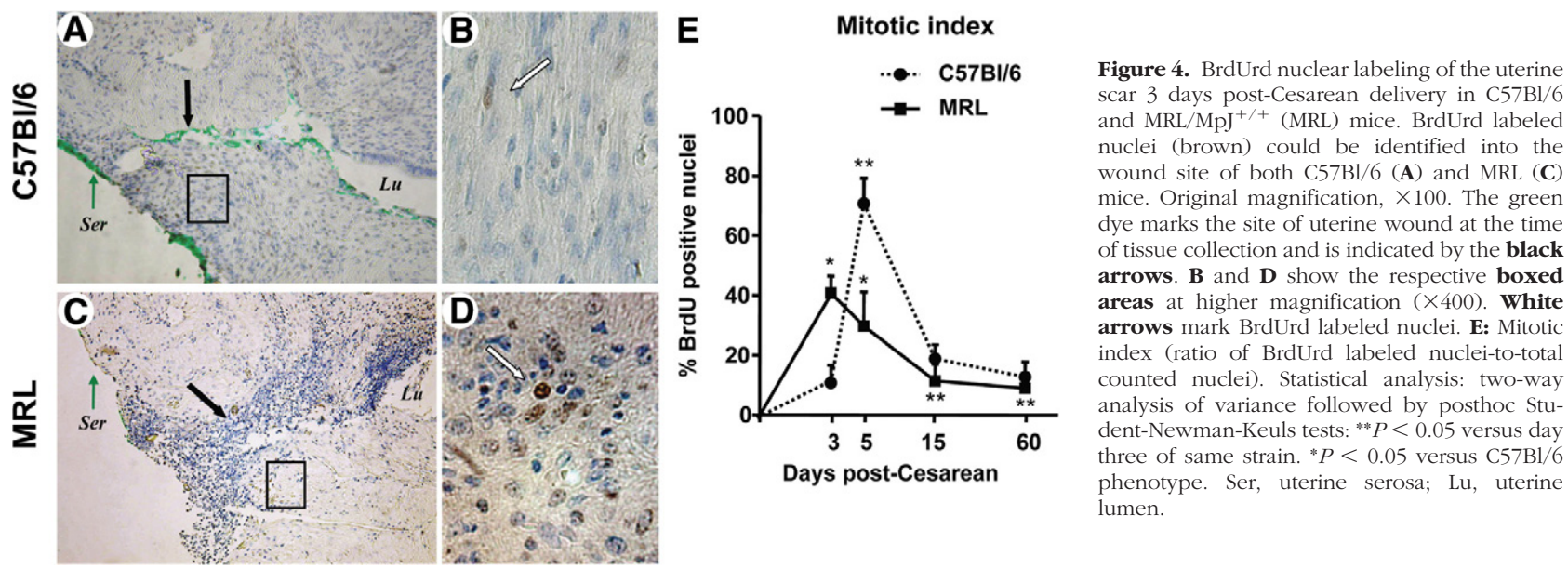

ure $3 \mathrm{D}, \mathrm{H}$, and $\mathrm{L}$ ). The fibrinous exudate was absent and there were no stitches visible. The circular muscle was restored around the intact lumen and the serosa returned to normal as shown by the continuous birefringent external layer (Figure 3L). Conversely, in the C57BL/5 mice the surgical stitches were still visible in 3 of 5 animals. The site of the scar was recognized as a thinner tissue integration layer (Figure 3C, G, and K) with discontinuous birefringent serosa (Figure 3K). Collectively, these results suggest an accelerated collagen maturation process in the MRL strain.

\section{BrdUrd Detection at the Site of Uterine Scar}

As shown, we could recognize BrdUrd positive nuclei in the uterine scar area beginning with day 3 after surgery (C57BI/6: Figure 4, A and B; MRL: Figure 4, C and D). These data confirm that healing of a hysterotomy implies an initial phase characterized by involvement of mitotically active cells. Three days after injury, more BrdUrd labeled nuclei were visualized into the wound site of the MRL compared with C57BI/6 mice. In the MRL mice, mitotic activity was highest on day 3 postsurgery compared with all other analyzed days and mostly localized in the serosa and glands. Interestingly, serosa had many more BrdUrd positive nuclei than the myometrium as illustrated by the intensely stained serosa under the green tissue dye. The analysis of the mitotic index showed the most intense mitotic activity in the MRL strain on day 3 and day 5 compared with on days 15 and 60 (Figure 4E, two-way analysis of variance, $P<0.001$ ). On day 3 postsurgery the MRL mice had higher mitotic activity at the wound site compared with C57BI/6 $(P=$ 0.007). This was in contrast to day 5 when the mitotic activity peaked for C57BI/6 mice $(P<0.001)$. On days 15 and 60 , the overall wound mitotic activity decreased compared with days 3 and 5 in both strains. These results are consistent with a shift in mitotic activity toward the early postsurgical period in the "high healer" phenotype.

To determine the nature of proliferating cells during the healing process post-CD, we performed double immunohistochemistry for BrdUrd and $\alpha$-SMA, as marker of smooth muscle differentiation (Figure 5). The first obser- vation was that on day 3 post CD the vast majority of the BrdUrd positive cells were devoid of $\alpha$-SMA (Figure 5, A and $B$ ). In both strains these cells were preferentially located in the vicinity of the wound integration area between the bundles of myometrial smooth muscle fibers as also noted on day 5 (Figure 5, C-E). In contrast, on day 15 (Figure 5, G and $\mathrm{H}$ ) and more prominently on day 60 post-CD (Figure 5, I-K), we observed that most of BrdUrd positive nuclei belonged to cells that also stained positively for $\alpha$-SMA thus indicative of myogenic differentiation (Figure 5K). BrdUrd positive cells were also observed in the wall of dilated blood vessels as shown in Figure 5l. Although on days 15 and 60 post-CD, the mitotic index did not differ between C57BI/6 and MRL mice, it did appear that the most numerous $\mathrm{BrdUrd}^{+} / \alpha$ $\mathrm{SMA}^{+}$cells were localized in the subserosal area in C57BI/6 mice (Figure 5, G and I), whereas in MRL mice (Figure $5, \mathrm{H}$ and $\mathrm{J}$ ) a mixture of $\mathrm{BrdUrd}^{+} / \alpha-\mathrm{SMA}^{+}$and $\mathrm{BrdUrd}^{+} / \alpha-\mathrm{SMA}^{-}$cells were present throughout the healed area.

\section{Tensile Load Testing of the Uterus}

To demonstrate the role of genetic background on functional behavior of the uterine scar, stress-strain curves were generated on days 3,15 , and 60 postoperatively. The stiffness of $\mathrm{C} 57 \mathrm{BI} / 6$ scarred myometrium increased linearly (slope day 3: $4.8 \pm 0.6$ versus day 15: $10.6 \pm 1.9$ versus day 60: $18.3 \pm 3.3 \mathrm{~N} / \mathrm{mm} / \mathrm{Gram}$, one-way analysis of variance, $P<0.01$; Figure $6 \mathrm{~A}$ ). In contrast, the MRL scar reached its maximal stiffness on day 15 (slope day 3: $1.05 \pm 0.5$ versus day $15: 8.7 \pm 2.7 \mathrm{~N} / \mathrm{mm} / \mathrm{Gram}, P=$ 0.007 ) and then plateaued at a lower level than the C57BI/6 (slope day $60 \mathrm{C} 57 \mathrm{BI} / 6: 33.1 \pm 3.5$ versus MRL $8.9 \pm 0.9 \mathrm{~N} / \mathrm{mm} / \mathrm{Gram}$, two-way analysis of variance, $P=$ 0.01 ). At 60 days post-CD, the MRL mice displayed significantly lower yield point compared with C57BI/6 phenotype (two-way analysis of variance, $P=0.01$ for strain, $P=0.02$ for days; Figure $6 \mathrm{~B}$ ). The BP evolved similarly in the two mouse strains. Less tissue displacement was required to reach $B P$ on days 15 and 60 , in both $M R L$ and C57BI/6 strains compared with day 3 (two-way analysis of variance, $P<0.001$; Figure $6 C$ ). To summarize, the un- 
C57BI/6
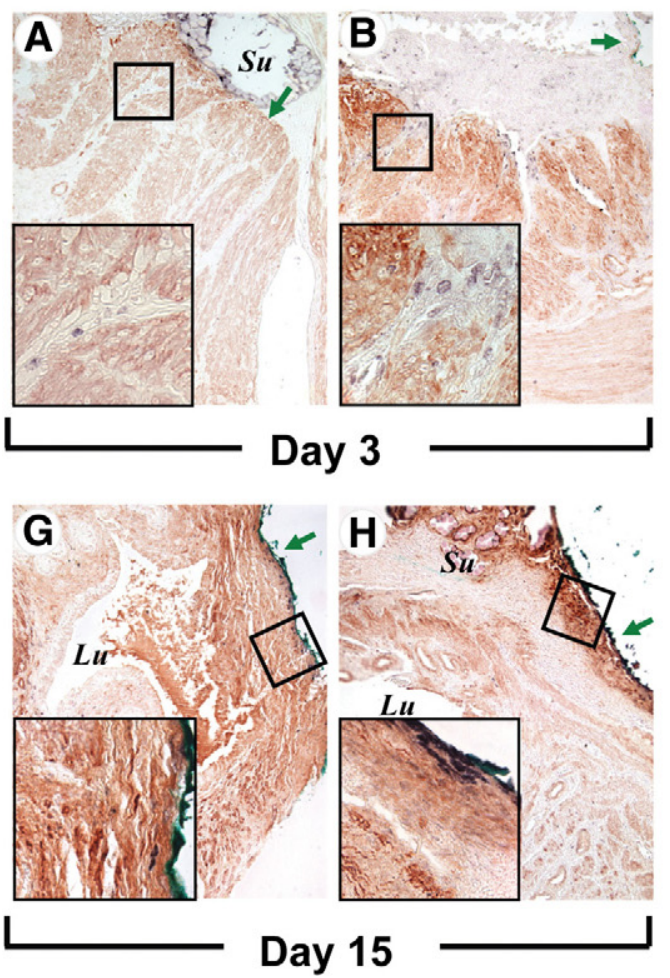

C57BI/6

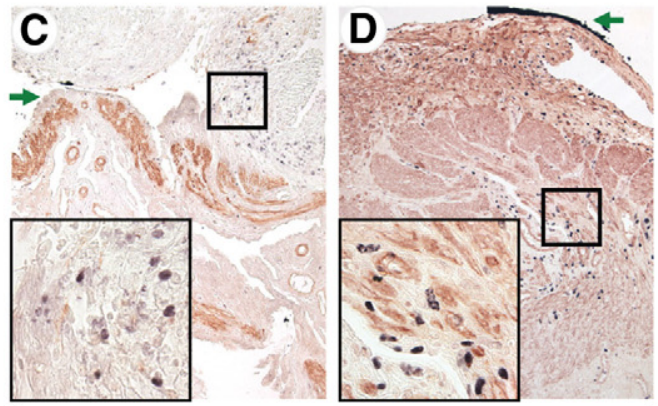

Day 5

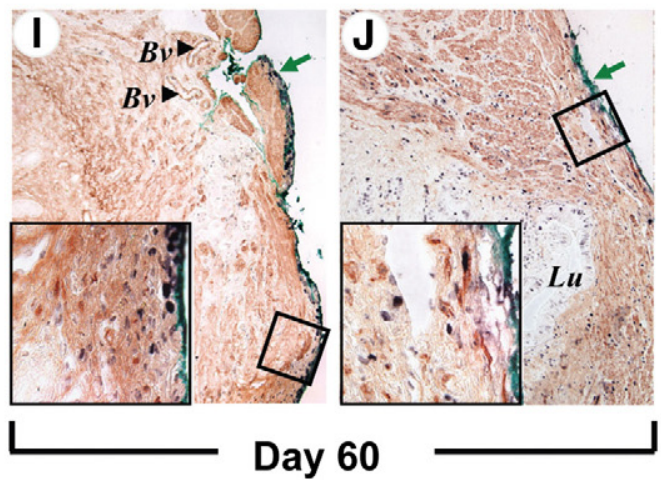

MRL

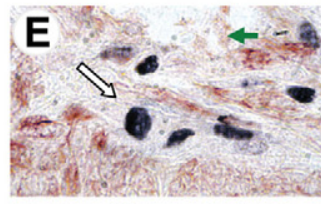

$\mathbf{F}$

Su

Figure 5. Topographical co-localization of BrdUrd and smooth muscle actin post-Cesarean delivery in C57Bl/6 and MRL/MpJ ${ }^{+/+}$(MRL) mice. Cells with BrdUrd labeled nuclei (purple black) could be identified between the red muscle bundles in C57Bl/6 [day 3 (A) and day 5 (C)] and MRL [MRL: day 3 (B) and day five (D)] mice. At the later times, cells positive for both BrdUrd and smooth muscle actin could be observed in proximity to serosa in the scar area both in $\mathrm{C} 57 \mathrm{Bl} / 6$ [day $15(\mathbf{G})$ and day $60(\mathbf{I})$ ] and MRL [MRL: day $15(\mathbf{H})$ and day $60(\mathbf{J})$ ] mice. Original magnification, $\times 100$ (Scale bar $=100 \mu \mathrm{m})$. The areas delineated by the squares are further shown in the left lower corner of each panel as higher magnification captions of the boxed areas $(\times 640 ;$ Scale bar $=33 \mu \mathrm{m})$. E and $\mathbf{K}$ show representative areas at $\times 1000$ magnification of $\mathbf{D}$ (day five) and $\mathbf{J}$ (day 60) in MRL mice. F shows a section from an MRL animal (day five) processed identically but omitting the two primary antibodies (Scale bar $=100 \mu \mathrm{m}$ ). The green arrows indicate the site of the uterine scar as marked with tissue dye (green or black). The white arrow in $\mathbf{K}$ points to a BrdUrd ${ }^{+} / \alpha-\mathrm{SMA}^{+}$cell in the subserosal area of regenerated uterine wall. Su, suture material; Lu, uterine lumen; Bv, blood vessel (arrowhead).

derlying regenerative ability of MRL and C57BI/6 mice translates in functional differences in the tensile viscoelastic properties of their scarred myometrium after CD. Similar BPs suggests that myometrial strength post-CD is not influenced by the underlying healing phenotype. The ruptures of the mounted uterine rings occurred at either the scar's site or near the scar in the zone of transition to the uninjured uterine wall. On day 3 , most uterine rings broke at the scar's site in both strains (scar rupture $78 \%$ [7 of 9]), whereas on day 15 , uterine ring ruptures occurred most often near the scar in both strains (scar rupture $25 \%$ [2 of 8 ], day 3 versus day 15, Fisher's exact, $P=0.057$ ). Interestingly, on day 60 , most ruptures occurred again at the site of the scar (scar rupture $70 \%$ [7of 10]).

\section{Discussion}

The results outlined in this study suggest that healing and tensile visco-elastic behavior of the surgically wounded myometrium varies with the genetic background and is phenotype dependent. By using two strains of mice that differ in their underlying regenerative healing and colla-

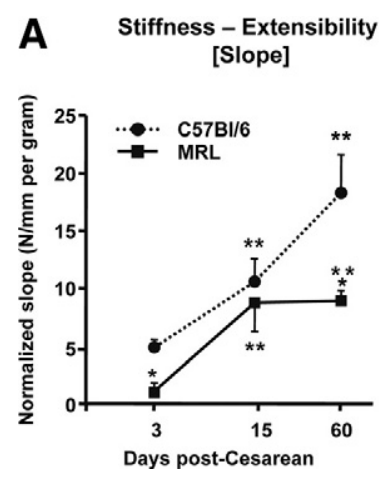

B

Elasticity - Plasticity [Yield Point]

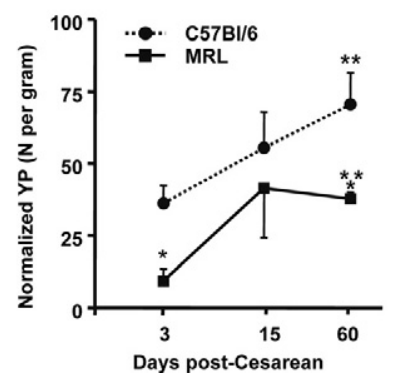

\section{Strength - Weakness [Break Point]}

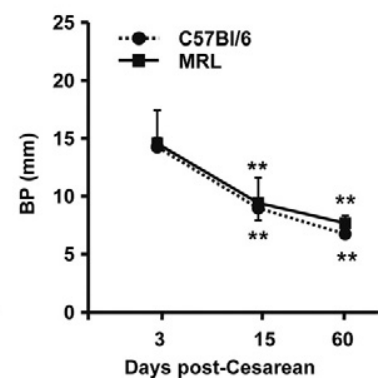

Figure 6. Analysis of the load-displacement relationships of scarred uterine rings in $\mathrm{C} 57 \mathrm{Bl} / 6$ and $\mathrm{MRL} / \mathrm{MpJ}^{+/+}$(MRL) mice. Uterine rings were collected at 3,15 , and 60 days post-Cesarean delivery and demonstrated differences in the slope (A: measure of stiffness), yield point (YP B: measure of plasticity), and break point (BP, $\mathbf{C}$ : measure of strength). Statistical analysis: twoway analysis of variance followed by posthoc Student-Newman-Keuls tests. ${ }^{* * *} P<0.05$ versus day 3 of same strain. ${ }^{*} P<0.05$ versus $\mathrm{C} 57 \mathrm{Bl} / 6$ phenotype. 
gen remodeling characteristics, ${ }^{11}$ we demonstrated significant differences in the initial but not late macroscopic aspect of myometrial wound healing process. We have also shown that the process of wound integration, inflammation, course of collagen synthesis, maturation of the myometrial wound, and the involvement of mitotic active cells in the initial stages of myometrial healing is phenotype dependent. The results of our study suggest that after $C D$ the underlying regenerative ability of the "high healer" phenotype (MRL) translates in functional differences in tensile visco-elastic properties of the scarred myometrium. The mechanical strength of the uterine scar did not appear to be phenotype dependent as the end result for the scarred tissue BP was similar in the MRL and $\mathrm{C} 57 \mathrm{BI} / 6$ mice, 60 days postdelivery. Finally, we have shown that rupture of the surgically wounded myometrium may occur at either the scar's site or near the scar, in the zone of transition to the uninjured uterine wall. Thus, our hypothesis that the inborn wound remodeling phenotype impacts on the process of myometrial wound healing and determines the future morphology and functional behavior of the uterine scar was confirmed.

Our research was motivated by previous studies that have demonstrated that although $60 \%$ to $80 \%$ of women who attempt vaginal birth after CD are ultimately successful, the risk of potentially catastrophic uterine rupture exceeds $1 \% .{ }^{23}$. Therefore, the enthusiasm for a trial of labor after a prior $\mathrm{CD}$ has considerably diminished because of safety concerns. Traditionally, an increased uterine contractility during labor and the surgical technique used for closure of the hysterotomy were thought to be the primary causes of uterine rupture. ${ }^{24,25}$ However, although all of this may hold true, the "burst" of the lower uterine segment during quiescent phases of gestation or after either one- or two-layer closure of the uterine incision suggest that structural defects after impaired surgical healing may also contribute to rupture. ${ }^{26}$

The healing process of a human CD scar is still subject to controversy and intense speculation. ${ }^{27}$ Beginning with Kroenig's ${ }^{28}$ description of the lower uterine segment incision, physicians have debated about scar formation in the uterine wall. ${ }^{28,29}$ Williams $^{2}$ opined in 1921 that the uterus healed by regeneration of the muscular cells and that scarring appeared only in cases healing by secondary repair. Repina ${ }^{3}$ noted the findings of investigators who could find no scar in $54 \%$ of cases. In $29 \%$, they found a partial regeneration, and in $17 \%$ they found only connective tissue. Since the original description, most authors have believed that the repairing of the muscular tissue occurred by scar formation. ${ }^{30,31}$ Taken together, these observations have led to the conclusion that the healing process of human myometrium is subject to large variance. Along with the number of prior $\mathrm{CDs}$, one possible explanation for the presence or absence of an identifiable uterine scar may rest with the genetic makeup. Because wound repair is a genetically controlled process requiring the concerted activity of cells involved in inflammation, hemostasis, apoptosis, immune defense, regeneration, tissue remodeling, and epithelialization, each of these steps could lead to a different or an altered healing process. ${ }^{16}$
In the past we proposed that the process of myometrial wound healing impacts on the future morphology and functional behavior of the uterine scar. ${ }^{32}$ For obvious reasons, in humans, difficulties in obtaining serial samples of the uterine scar are a major barrier to our understanding of the events involved in the reparative process. Therefore, development of animal models to allow for a better understanding of the healing process of mammalian tissue is critical. ${ }^{33}$ The MRL mouse model was previously used to study the biology of the ear and heart wound repair. ${ }^{10,11,13}$ The advantage of this unique MRL mouse is that this phenotype has an exaggerated wound healing regenerative response to injury. Thus, the MRL healing process can be directly compared with that of mouse strains that are incapable of regenerating. ${ }^{15,16}$ Our study applied, apparently for the first time, the MRL model to the study of the process of uterine healing post-CD. In the course of this work, it became apparent that the MRL and C57BI/6 mice differ in various aspects of postpartum healing. Although wound healing of the myometrium and the ear can be subject to different controlling mechanisms, the observed differences in closure of the ear after wounding argues that our animal model was appropriate.

Uterine wound repair involves two major issues: the rate and the quality of the healing process. The results presented here provide evidence that the uterine wound is filled with vascular granulation tissue at 3 to 5 days postsurgery. Yet the process of angiogenesis and blood vessels disintegration secondary to apoptosis may occur at a different pace in the two strains because at 5 days post-CD granulation tissue buds were still present in C57BI/6 but not in the MRL mice. Interestingly, the site of the uterine scar could be identified macroscopically in both strains by 15 but not by 60 days postsurgery. This may explain why in humans the uterine scar may be recognized after a short interpregnancy interval. Hence, visualization of the uterine scar in humans may be dependent on the duration of the myometrial wound remodeling process. ${ }^{34}$

The debate on the role of surgical closure technique in lowering the risk of uterine rupture is not over, and our findings may have clinical relevance in this regard. In our animal model, the presence of lax surgical stitches only days after surgery was a consistent finding among animals. This argues that the surgical closure technique is not the only element that defines the quality of the uterine wound healing. Due to the normal process of uterine tissue involution in postpartum and our macroscopic findings, it is reasonable to assume that the tensile load in the stitch decreases in the first few days after surgery. Therefore, the main purpose of the stitches could be to ensure hemostasis and correct anatomical apposition of the myometrial tissue in the immediate postsurgical phase. Once wound integration occurs (which appears to be phenotype dependent), the mechanical function of the stitches becomes unnecessary. Importantly, our observation that tissue disruption did not always occur at the site of uterine scar but also in the zone of transition to the uninjured myometrium suggests that changes in surgical closure techniques may prevent subsequent uterine ruptures only to the 
extent where the architecture of the post-CD uterine wall is restored to normal. Further studies remain to provide a definitive answer to these important issues.

Histological examination of the uterine healing process demonstrated significant differences in scar integration, level of inflammation, and collagen remodeling at the site of myometrial injury between the MRL and C57BI/6 mice. Although these processes were present in both strains of mice, their length, window of occurrence, and duration varied. Thus, it is plausible that the timing of inflammation, collagen deposition, and remodeling process of the uterine scar are genetically programmed. Although our study does not prove causality, it suggests that the sequence of events involved in the initial phase of myometrial wound healing may be responsible for the observed differences in the stiffness and elasticity of the uterine scar. Interestingly, we should point out that the scarred uterine rings break at similar extensions in both phenotypes. At this time it is still unknown if a longer period of observation would have demonstrated significant differences in tissue strength between strains or if the scar's resistance to stretch would behave similarly in a subsequent pregnancy. Prior animal studies have demonstrated that wound healing is a polygenically determined quantitative trait with an estimated heritability of $86 \% .{ }^{35}$ Further studies should be performed to provide insight on whether the sequence of events, intensity of different phases of wound healing, and tensile visco-elastic properties of the uterine scar can be reversed or modified by using F2 populations of MRL and $\mathrm{C} 57 \mathrm{BI} / 6$ crosses.

An important finding of our study is that 60 days after surgery, the MRL wound architecture was restored to normal. Our analysis of collagen deposition by polarized microscopy was not able to recognize the site of the prior uterine hysterotomy as a distinct collagen birefringent area. This is a significant finding because in most mammal tissues collagen remains the most critical element responsible for maintenance of tissue structural integrity. ${ }^{36}$ First, our results seem to suggest that at least after one CD collagen deposition is not the primary healing mechanism. Second, the information gained from this investigation implies that injured myometrial sites display mitotic activity. We showed that 3 days after surgery the most intense mitotic activity was present in the "high healer" phenotype and that serosa had much more BrdUrd positive nuclei than the myometrium. Conversely, mitotic activity peaked only later (day 5) in the $\mathrm{C} 57 \mathrm{BI} / 6$ animals. Fifteen days after $\mathrm{CD}$, mitotically active cells were present but reduced in both $M R L$ and C57BI/6 mice. The presence of most of the BrdUrd positive nuclei at uterine surface and not at deep myometrial level confirms the high regenerative ability of the serosa and suggests that the evidence necessary to confirm or disprove the proliferative ability of the uterine myocyte should be further sought.

Our study provides evidence that several population of cells proliferate in the injured area of which a subset eventually acquire $\alpha$-SMA and thus commit to uterine smooth muscle differentiation. These results should be placed in the context of the current knowledge of the molecular and cellular processes involved in regeneration of muscular fibers. ${ }^{37}$ For example, several types of progenitor cells have been found to play critical roles in the repair of injured skeletal, cardiac, or vascular smooth muscle. ${ }^{37}$ Out of the progenitor cell family, satellite cells are a population of tissue resident progenitors committed to myogenic differentiation that reside beneath the basal lamina of mature myofibers. ${ }^{38}$ Satellite cell-mediated repair of muscle fibers is critical for recovery of muscle function after suppression of inflammation. ${ }^{38} \mathrm{~A}$ sequence of well defined molecular signals results in activation of dormant satellite cells, proliferation, myogenic differentiation, and fusion with damaged muscle fibers. ${ }^{38}$ Additionally, studies in mouse models have determined that bone marrow-derived multilineage stem cells also have the ability to integrate into regenerating myofibers contingent on the microenvironment. ${ }^{39}$ At this time there is no published data with respect to the extent with which either satellite or hematopoetic stem cells participate in the repair of the uterus post-CD. Further research is needed to provide answers about the relationships among genetic background, participation of the various pluripotent niches in the process of uterine healing, and the myometrium's ability to functionally recover in a subsequent pregnancy.

A direct comparison between humans and animals is complex. The period of gestation for the mouse is 20 days, whereas that for the human is 40 weeks. ${ }^{40}$ Therefore, each day of gestation for the mouse is approximately equivalent to 2 weeks of gestation for the human. Extrapolating this to the postpartum period, we derive that day 3 post-CD in our mouse model could represent approximately 6 weeks human postpartum; day $5=10$ weeks postpartum; day $15=$ 30 weeks ( 7.5 months) postpartum; and day $60=2.5$ years postpartum. These intervals may have significant clinical relevance to humans. Epidemiological studies demonstrated that women who attempt vaginal birth after CD at less than 2 years post-CD have a higher risk of uterine rupture. $^{41}$ As we previously proposed, this observation could be related to phenotypical differences in the quality of the myometrial wound healing. ${ }^{32,34}$ Indeed, the results of the current study suggest that this may be the case. In our mouse model, the site of the uterine scar was active and underwent significant remodeling transformation even at 15 days post-CD (7.5 months in human equivalent). These changes were phenotypically dependent. In addition, several biomechanical endpoints differed between strains even 60 days post-CD (2.5 years in human equivalents). Therefore, our study provides indirect biological evidence that the human uterine scar could be a site of active remodeling, which may extend well beyond into postpartum. Development of noninvasive techniques that may asses the quality of the uterine healing process in humans for optimal timing of a new pregnancy in each individual is critically needed.

\section{References}

1. Hamilton BE, Martin JA, Ventura SJ: Births: preliminary data for 2007 Natl Vital Stat Rep 2009, 57:1-23

2. Williams JW: A critical analysis of 21 years experience with cesarean section. Bull Johns Hopkins Hosp 1921, 32:173-190

3. Repina MM: Case reports on uterine rupture in pregnancy after cesarean section. Akush Ginekol (Mosk) 1955, 6:61-62 
4. Goss RJ, Grimes LN: Epidermal downgrowths in regenerating rabbit ear holes. J Morphol 1975, 146:533-542

5. Darby IA, Hewitson TD: Fibroblast differentiation in wound healing and fibrosis. Int Rev Cytol 2007, 257:143-179

6. Stocum DL: The urodele limb regeneration blastema: determination and organization of the morphogenetic field. Differentiation 1984 . 27:13-28

7. Martin P: Wound healing: aiming for perfect skin regeneration. Science 1997, 276:75-81

8. Cass DL, Adzick NS: Fetal wound repair renaissance. Oral Maxillofacial Surg Clin 1996, 8:535-546

9. Singer AJ, Clark AF: Cutaneous wound healing. N Engl J Med 1999 341:738-746

10. Heber-Katz E: The regenerating mouse ear. Semin Cell Dev Biol 1999, 10:415-419

11. Clark LD, Clark RK, Heber-Katz E: A new murine model for mammalian wound repair and regeneration. Clin Immunol Immunopathol 1998, 88:35-45

12. Gourevitch D, Clark L, Chen P, Seitz A, Samulewicz SJ, Heber-Katz E: Matrix metalloproteinase activity correlates with blastema formation in the regenerating MRL mouse ear hole model. Dev Dyn 2003, 226:377-387

13. Leferovich JM, Bedelbaeva K, Samulewicz S, Zhang XM, Zwas D, Lankford EB, Heber-Katz E: Heart regeneration in adult MRL mice. Proc Natl Acad Sci USA 2001, 98:9830-9835

14. Heber-Katz E, Gourevitch D: The relationship between inflammation and regeneration in the MRL mouse: potential relevance for putative human regenerative (scarless wound healing) capacities? Ann NY Acad Sci 2009, 1172:110-114

15. McBrearty BA, Clark LD, Zhang XM, Blankenhorn EP, Heber-Katz E: Genetic analysis of a mammalian wound-healing trait. Proc Natl Acad Sci USA 1998, 95:11792-11797

16. Li X, Mohan S, Gu W, Baylink DJ: Analysis of gene expression in the wound repair/regeneration process. Mammalian Genome 2001, 12:52-59

17. Buhimschi CS, Gokdeniz R, Saade GR, Buhimschi IA, Garfield RE: The effect of chronic nitric oxide synthase inhibition on blood pressure and heart rate in unrestrained pregnant rats as recorded by radiotelemetry. Am J Obstet Gynecol 1999, 181:159-164

18. Buhimschi CS, Sora N, Zhao G, Buhimschi IA: Genetic background affects the biomechanical behavior of the postpartum mouse cervix. Am J Obstet Gynecol 2009, 200:434.e1-e7

19. Junqueira LC, Bignolas G, Brentani RR: Picrosirius staining plus polarization microscopy, a specific method for collagen detection in tissue sections. Histochem J 1979, 11:447-455

20. Hayashi Y, Koike M, Matsutani M, Hoshino T: Effects of fixation time and enzymatic digestion on immunohistochemical demonstration of bromodeoxyuridine in formalin-fixed, paraffin-embedded tissue. J Histochem Cytochem 1988, 36:511-514

21. Buhimschi IA, Dussably L, Buhimschi CS, Ahmed A, Weiner CP: Physical and biomechanical characteristics of rat cervical ripening are not consistent with increased collagenase activity. Am J Obstet Gynecol 2004, 191:1695-1704

22. Buhimschi CS, Buhimschi IA, Yu C, Wang H, Sharer DJ, Diamond MP, Petkova AP, Garfield RE, Saade GR, Weiner CP: The effect of dystocia and previous cesarean uterine scar on the tensile properties of the lower uterine segment. Am J Obstet Gynecol 2006, 194:873-883
23. Lydon-Rochelle M, Holt V, Easterlig TR, Martin DP: Risk of uterine rupture during labor among women with a prior cesarean delivery. N Engl J Med 2001, 345:3-8

24. Garnet JD: Uterine rupture during pregnancy: an analysis of 133 patients. Obstet Gynecol 1964, 23:898-905

25. Tucker JM, Hauth JC, Hodgkins P, Owen J, Winkler CL: Trial of labor after a one or two layer closure of a low transverse uterine incision. Am J Obstet Gynecol 1993, 168:545-546

26. Phelan JP, Korst LM, Settles DK: Uterine activity patterns in uterine rupture: a case-control study. Obstet Gynecol 1998, 92:394-398

27. Cunningham FG, Gant NF, Leveno KJ, Gilstrap LC, Hauth JC, Wenstrom KD: Obstetrical hemorrhage. In Williams Obstetrics. 21st ed. Columbus, $\mathrm{OH}$ The McGraw-Hill Co. Inc, 2001

28. Kronig B. Transperitonealer, cervikaler kaiserschnitt. In: Doderlein A, Kronig B, eds. Operative gynakologie. 3rd ed. Leipzig: Georg Thieme, 1912:870-886

29. Wojdecki J, Grynsztajn A: Scar formation in the uterus after cesarean section. Am J Obstet Gynecol 1970, 107:322-324

30. Nakonieczny Z: Conduction of labor following cesarean section Ginek Pol 1962, 33:251-258

31. Septien JM, Baunnet CF: Estudio histologico y valoracion de cicatrices uterinas consecutivas a diversos tipos de cesarean. Acta Ginecol (Madr) 1961, 12:449-456

32. Buhimschi CS, Buhimschi IA, Patel S, Malinow A, Weiner CP: Rupture of the uterine scar during term labor: contractility or biochemistry? BJOG 2005, 112:38-42

33. Schwartz OH, Paddock R: The cesarean scar. Am J Obstet Gynecol 1925, 10:153-171

34. Hamar BD, Saber SB, Cackovic M, Magloire LK, Pettker CM, AbdelRazeq SS, Rosenberg VA, Buhimschi IA, Buhimschi CS: Ultrasound evaluation of the uterine scar after cesarean delivery: a randomized controlled trial of one- and two-layer closure. Obstet Gynecol 2007, $110: 808-813$

35. Li X, Gu W, Masinde G, Hamilton-Ulland M, Xu S, Mohan S, Baylink DJ: Genetic control of the rate of wound healing in mice. Heredity 2001, 86:668-674

36. Leppert PC: Anatomy and physiology of cervical ripening. Clin Obstet Gynecol 1995, 38:267-279

37. Schabort EJ, Myburgh KH, Wiehe JM, Torzewski J, Niesler CU: Potential myogenic stem cell populations: sources, plasticity, and application for cardiac repair. Stem Cells Dev 2009, 18:813-830

38. Boonen $\mathrm{KJ}$, Post MJ: The muscle stem cell niche: regulation of satellite cells during regeneration. Tissue Eng Part B Rev 2008 , 14:419-431

39. Hollemann D, Budka H, Löscher WN, Yanagida G, Fischer MB Wanschitz JV: Endothelial and myogenic differentiation of hematopoietic progenitor cells in inflammatory myopathies. J Neuropathol Exp Neurol 2008, 67:711-719

40. Holladay SD, Smialowicz RJ: Development of the murine and human immune system: differential effects of immunotoxicants depend on time of exposure. Environ Health Perspect 2000, 108 Suppl 3:463-473

41. Stamilio DM, DeFranco E, Paré E, Odibo AO, Peipert JF, Allsworth JE, Stevens E, Macones GA: Short interpregnancy interval: risk of uterine rupture and complications of vaginal birth after cesarean delivery. Obstet Gynecol 2007, 110:1075-1082 